

\title{
Characterizing the Subcortical Structures in Youth with Congenital Heart Disease
}

\author{
(D) Fontes, (D). Courtin, (D).V. Rohlicek, (D). Saint-Martin, (D) G. Gilbert, (D). Easson, (D)A. Majnemer, (D) A. Marelli, \\ (D) M.M. Chakravarty, and (D). Brossard-Racine
}

\begin{abstract}
BACKGROUND AND PURPOSE: Congenital heart disease is a leading cause of neurocognitive impairment. Many subcortical structures are known to play a crucial role in higher-order cognitive processing. However, comprehensive anatomic characterization of these structures is currently lacking in the congenital heart disease population. Therefore, this study aimed to compare the morphometry and volume of the globus pallidus, striatum, and thalamus between youth born with congenital heart disease and healthy peers.
\end{abstract}

MATERIALS AND METHODS: We recruited youth between 16 and 24 years of age born with congenital heart disease who underwent cardiopulmonary bypass surgery before 2 years of age $(n=48)$ and healthy controls of the same age $(n=48)$. All participants underwent a brain MR imaging to acquire high-resolution 3D T1-weighted images.

RESULTS: Smaller surface area and inward bilateral displacement across the lateral surfaces of the globus pallidus were concentrated anteriorly in the congenital heart disease group compared with controls $(q<0.15)$. On the lateral surfaces of bilateral thalami, we found regions of both larger and smaller surface areas, as well as inward and outward displacement in the congenital heart disease group compared with controls $(q<0.15)$. We did not find any morphometric differences between groups for the striatum. For the volumetric analyses, only the right globus pallidus showed a significant volume reduction $(q<0.05)$ in the congenital heart disease group compared with controls.

CONCLUSIONS: This study reports morphometric alterations in youth with congenital heart disease in the absence of volume reductions, suggesting that volume alone is not sufficient to detect and explain subtle neuroanatomic differences in this clinical population.

ABBREVIATION: $\mathrm{CHD}=$ congenital heart disease

ongenital heart disease (CHD) is a leading cause of neurodevelopmental impairment. ${ }^{1}$ A variety of motor delays, language disorders, behavioral problems, and learning difficulties have been described in children and adolescents with $\mathrm{CHD}{ }^{2,3}$ Adults and senior citizens with CHD are also at a greater risk for neurocognitive challenges and early cognitive decline. ${ }^{4}$ Brain

Received March 4, 2020; accepted after revision May 19.

From the Advances in Brain and Child Health Development Research Laboratory (K.F., F.C., K.E., M.B.-R.), Centre for Outcomes Research \& Evaluation, Research Institute of the McGill University Health Centre, Montreal, Quebec, Canada; Department of Pediatrics, Division of Cardiology (C.V.R.), Department of Medical Imaging, Division of Pediatric Radiology (C.S.-M.), Department of Pediatrics, Division of Neurology (A. Majnemer), and Department of Pediatrics, Division of Neonatology (M.B.-R.), Montreal Children's Hospital McGill University Health Centre, Montreal, Quebec, Canada; MR Clinical Science (G.G.), Philips Healthcare, Markham, Ontario, Canada; School of Physical and Occupational Therapy (A. Majnemer, M.B.-R.), Departments of Psychiatry (M.M.C.), and Biological and Biomedical Engineering (M.M.C.), McGill University, Montreal, Quebec, Canada; McGill Adult Unit for Congenital Heart Disease Excellence (A. Marelli), McGill University Health Center, Montreal, Montreal, Quebec, Canada; and

Computational Brain Anatomy Laboratory (M.M.C.), Cerebral Imaging Centre,

Douglas Mental Health University Institute, Verdun, Quebec, Canada. injury is a frequent neonatal complication of $\mathrm{CHD}$, and recent studies suggest that up to $65.7 \%$ of adolescents born with $\mathrm{CHD}$ who underwent open heart surgery requiring cardiopulmonary bypass during infancy present with overt brain abnormal

This work was collectively funded by the Faculty of Medicine of McGill University and the McGill University Health Center. M.B.-R. hold a Fonds de recherche du Quebec-Sante Research Scholar award. K.F. received support from the McGill University Faculty of Medicine Internal Studentship Award and the Research Institute of the McGill University Health Center Desjardins Studentship in Child Health Research. Computations were performed on the Niagara supercomputer at the SciNet HPC Consortium. SciNet is funded by the Canada Foundation for Innovation under the auspices of Compute Canada, the Government of Ontario, Ontario Research Fund-Research Excellence program, and the University of Toronto.

Please address correspondence to Marie Brossard-Racine, OT, PhD, Centre for Outcomes Research \& Evaluation, Research Institute, McGill University Health Centre, 5252 Boulevard de Maisonneuve, 3F.46, Montréal, QC, H4A 3S5 Canada; e-mail: marie.brossardracine@mcgill.ca

Indicates article with supplemental on-line tables.

http://dx.doi.org/10.3174/ajnr.A6667 
findings on qualitative MR imaging. ${ }^{5}$ However, even in the absence of brain anomalies on qualitative MR imaging, adolescents with complex CHD often present with worse neurocognitive outcomes than healthy peers. ${ }^{5}$ Therefore, qualitative MR imaging may not capture subtle neuroanatomic differences potentially underlying neurodevelopmental deficits in this population.

Morphometry has the potential to characterize structural specificities that cannot be captured by volumetric analysis, such as bending, flattening, or focal surface area changes. ${ }^{6}$ In fact, morphometry has been shown to provide a sensitive marker for abnormal brain development in a number of neurodevelopmental and psychiatric disorders, including childhood-onset schizophrenia ${ }^{7}$ and autism spectrum disorder. ${ }^{8}$ Preliminary studies in adolescents with CHD have reported smaller basal ganglia nuclei in adolescents with CHD compared with controls. ${ }^{9,10}$ However, most of these volumetric differences were no longer apparent when controlling for overall brain volume differences. One recent study using whole-brain voxel-based morphometry found reduced gray matter density in many cortical regions of the brain, as well as in the thalamus, caudate nuclei, and putamen of adolescents with singleventricle CHD. ${ }^{11}$ However, comprehensive evaluation of the subcortical structures in youth with CHD using a hypothesisdriven approach is still missing. In a prior study, we successfully performed volumetric and morphometric analyses of the hippocampus and found differences between youth with CHD and healthy volunteers that were associated with poorer working memory. ${ }^{12}$ As a next step, we aimed, with this study, to quantitatively compare the morphometry and volume of the globus pallidus, striatum, and thalamus between youth with CHD and healthy peers. We hope that a comprehensive anatomic evaluation of these subcortical structures that play critical roles in many learning and cognitive processes ${ }^{13-15}$ will help provide a better understanding of the neural correlates of some of the neurocognitive difficulties observed in this population.

\section{MATERIALS AND METHODS \\ Participants}

We recruited term-born ( $>36$ weeks gestational age) youth, 1624 years of age, who underwent open heart surgery using cardiopulmonary bypass for complex CHD before 2 years of age and without documented congenital infection, chromosomal abnormalities, and/or multiorgan dysmorphic conditions. A control group of youth matched for age and sex was also recruited from the local community using pamphlets and social media. Controls were considered healthy if they had no history of brain tumor or malformation, traumatic brain injury, or developmental or neurologic conditions and had not undergone rehabilitation or received special education services during childhood or adolescence. Written informed consent was obtained from the participants or legal guardians when participants were younger than 18 years of age. All participants underwent brain MR imaging at the Montreal Children's Hospital. Socioeconomic status was evaluated using the Hollingshead Four Factor Index. ${ }^{16}$ The study was approved by the McGill University Health Center Pediatric Research Ethics Board.

\section{MR Imaging}

Image Acquisition. Participants were scanned on a 3T MR imaging system (Achieva X-Series; Philips Healthcare) using a 32-channel head coil to acquire 3D 1-mm isotropic anatomic T1-weighted images $(\mathrm{TE}=3.7 \mathrm{~ms}, \mathrm{TR}=8.1 \mathrm{~ms}, \mathrm{FOV}=240 \times$ $240 \times 180 \mathrm{~mm}$, section thickness $=1 \mathrm{~mm}$, flip angle $\left.=8^{\circ}\right)$ of their brains. All images were qualitatively reviewed for brain abnormalities by an experienced neuroradiologist (C.S.-M.) blinded to the participant's medical history.

Image Analysis or Image Processing. All acquired images underwent visual quality assessment and were excluded if excessive motion or scanner artifacts were observed. Images were visually inspected for quality and segmentation accuracy at each processing step. ${ }^{17}$ Images were first preprocessed using the mincbpipe-library (https://github.com/CobraLab/minc-bpipe-library) ${ }^{18}$ This pipeline uses a "clean_and_center" stage to uniformize the direction cosines and the zero-point of the scan to the center of the image, followed by bias field correction using the N4ITK algorithm (https://simpleitk.readthedocs.io/ en/master/link_N4BiasFieldCorrection_docs.html), ${ }^{19}$ brain extraction based on nonlocal segmentation technique, ${ }^{20}$ and image cropping to remove the neck. Total brain volume estimates were acquired from the brain extraction based on the nonlocal segmentation technique mask. ${ }^{20}$

The MAGeT-Brain algorithm (https:/github.com/CobraLab/ MAGeTbrain) is an automatic segmentation ${ }^{21}$ that has been validated in healthy and clinical populations of various ages and provides quickly accessible, reliable, and reproducible results. ${ }^{21-25}$ This algorithm uses a multiatlas voting method, based on a template library generated from a subset of the sample, to segment images while incorporating neuroanatomic variation specific to the population of interest. Surface-based measures of displacement are measurements of the deformation along the normal at each vertex of surface-based models of each structure as estimated by calculating the dot product between the normal and the average deformation vector at that point. Local surface area is estimated as the mean surface of all adjacent triangles after deformation with the mean deformation field and reparcellation scheme using Vornoi mapping (http://webhelp.esri.com/ arcgisdesktop/9.2/index.cfm?TopicName=Voronoi_maps). All preprocessed images were entered into the MAGeT-Brain pipeline to delineate the thalamus, striatum, and globus pallidus (Fig 1). Outputs from the MAGeT-Brain pipeline included volumes, surface area, and displacement measurements for each structure. All computations were performed on the Scinet University of Toronto cluster (https://www. scinethpc.ca/). ${ }^{26}$ Vertex-wise morphometry analyses were then conducted through the RMINC library in R (Version 3.4.4; http://www.r-project.org). ${ }^{27,28}$

\section{Statistical Analysis}

Participants' characteristics were compared between groups using $\chi^{2}$ tests for categoric variables and independent $t$ tests for 


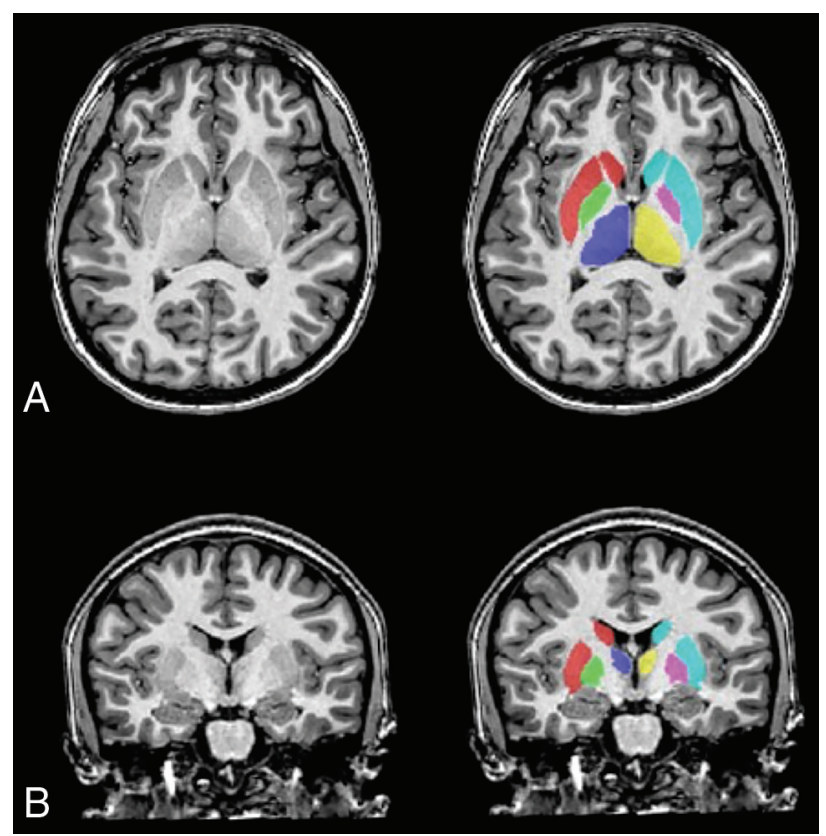

FIG 1. Segmentation of the subcortical structures. T1-weighted image of a female participant with CHD (17 years of age) with delineation of the subcortical structures: globus pallidus left (green) and right (fuschia), thalamus left (blue) and right (yellow), and striatum left (red) and right (turquoise) in the axial $(A)$ and coronal $(B)$ planes.

continuous variables, which were confirmed to be normally distributed using Shapiro-Wilk tests. Subcortical morphometric and volumetric differences between groups were assessed using the FSL General Linear Model (http://fsl.fmrib.ox.ac.uk/fsl/ fslwiki/GLM). Variables significantly different between the groups were added to the GLMs as covariates. Post hoc significance testing with the false discovery rate method was performed for all GLMs to account for multiple comparisons using a threshold of $q<0.15$. All statistical analyses were completed using R (Version $3.4 .4)^{28}$

\section{RESULTS}

\section{Participant Characteristics}

A total of 48 participants with CHD (20/48 male; mean age, 20.1 years) and 48 controls (20/48 male; mean age 20.5 years) were included in this study. Twenty-two participants with CHD were recruited from a previous study, the Determinants of Active Involvement in Leisure for Youth (DAILY) study, ${ }^{2}$ and 26 were prospectively enrolled at our center. There were no significant differences with respect to age, sex, body mass index, or socioeconomic status between the DAILY participants and the newly enrolled participants. The participants' characteristics are presented in the Table. Socioeconomic status was significantly higher in the control group compared with CHD group.

The CHD group was predominantly composed of youth with a bivenontricular physiology $(37 / 48,77.1 \%)$, including destrotransposition of the great arteries $(n=18)$, tetralogy of Fallot $(n=$ $11)$, total anomalous pulmonary venous connection $(n=3)$, ventricular and atrial septal defects $(n=3)$, and truncus arteriosus type I $(n=2)$. Only $11 / 48(22.9 \%)$ participants presented with
Participants' characteristics

\begin{tabular}{lccr}
\hline $\begin{array}{c}\text { Variables; mean } \pm \text { standard } \\
\text { deviation, } \boldsymbol{n}(\%), \text { median } \\
\text { [range] }\end{array}$ & $\begin{array}{c}\text { CHD } \\
(\boldsymbol{n}=\mathbf{4 8})\end{array}$ & $\begin{array}{c}\text { Control } \\
(\boldsymbol{n}=\mathbf{4 8})\end{array}$ & $\begin{array}{c}\boldsymbol{P} \\
\text { value }\end{array}$ \\
\hline $\begin{array}{l}\text { Age at MRI, years } \\
\text { Sex }\end{array}$ & $20.16 \pm 2.2$ & $20.48 \pm 2.0$ & .458 \\
$\quad$ Male & $20(41.7 \%)$ & $20(41.7 \%)$ & \\
$\quad$ Female & $28(58.3 \%)$ & $28(58.3 \%)$ & \\
BMI & $23.59 \pm 4.78$ & $23.55 \pm 3.8$ & .965 \\
Socioeconomic status ${ }^{a}$ & $38.20 \pm 8.48$ & $44.30 \pm 6.9$ & $<.001$ \\
Type of CHD & & & \\
$\quad$ Univentricular & $11 / 48(22.9 \%)$ & & \\
Biventricular & $37 / 48(77.2 \%)$ & & \\
\hline
\end{tabular}

Note:-BMI indicates body mass index.

a Score on the Hollingshead Four-Factor Index of Social Status, with higher scores indicating higher social status.

univentricular physiology. The univentricular physiologies present in our sample were pulmonary atresia $(n=4)$, double-outlet right ventricle $(n=3)$, double-inlet left ventricle $(n=2)$, hypoplastic left-heart syndrome $(n=1)$, and Ebstein pulmonary atresia $(n=1)$.

\section{Qualitative MR Imaging Findings}

Brain abnormalities on qualitative MR imaging were more frequent in youth with $\mathrm{CHD}(22.9 \%$; 11/48) compared with controls (10.4\%; 5/48). However, this difference did not reach statistical significance $\left[\chi^{2}(1, n=96)=2.700, P=.085\right]$. The details of these brain abnormalities are reported in On-line Table 1. Brain abnormalities were all considered mild and were divided into two categories according to their probable origin as either acquired (eg, injury, volume loss) or developmental (eg, heterotopia, malformation). Overall, acquired abnormalities ( $\mathrm{CHD}, n=9$; controls, $n=3$ ) were more frequent than developmental abnormalities (CHD, $n=6$; controls, $n=2$ ). There were no significant differences in sex, age, socioeconomic status, or other clinical characteristics between $\mathrm{CHD}$ and control participants with and without brain abnormalities.

\section{Subcortical Volumes and Shape Analysis}

All images passed quality assessment and were included for analyses. Total brain volume and socioeconomic status were significantly lower in the $\mathrm{CHD}$ group and were therefore included in the General Linear Model analyses. Youth with CHD presented with a smaller left surface area of the globus pallidus concentrated anteriorly (Fig $2 A$ ) and bilateral inward displacement across the lateral surfaces (Fig $3 A$ ). In the CHD group, regions of both increased and decreased surface area were found on the lateral surfaces of the thalamus bilaterally (Fig 2B), as well as inward and outward displacement (Fig 3B) compared with controls. We did not find any significant morphometric differences between groups for the striatum. Last, there were no morphologic differences between the participants with or without brain abnormalities.

Youth with CHD had significantly smaller right globus pallidus volume than controls $(F(1,93)=4.867, q=0.036)$. There were no other volumetric differences between CHD participants and controls. We additionally performed volumetric comparison of the striatum once subdivided into the left and right putamen 


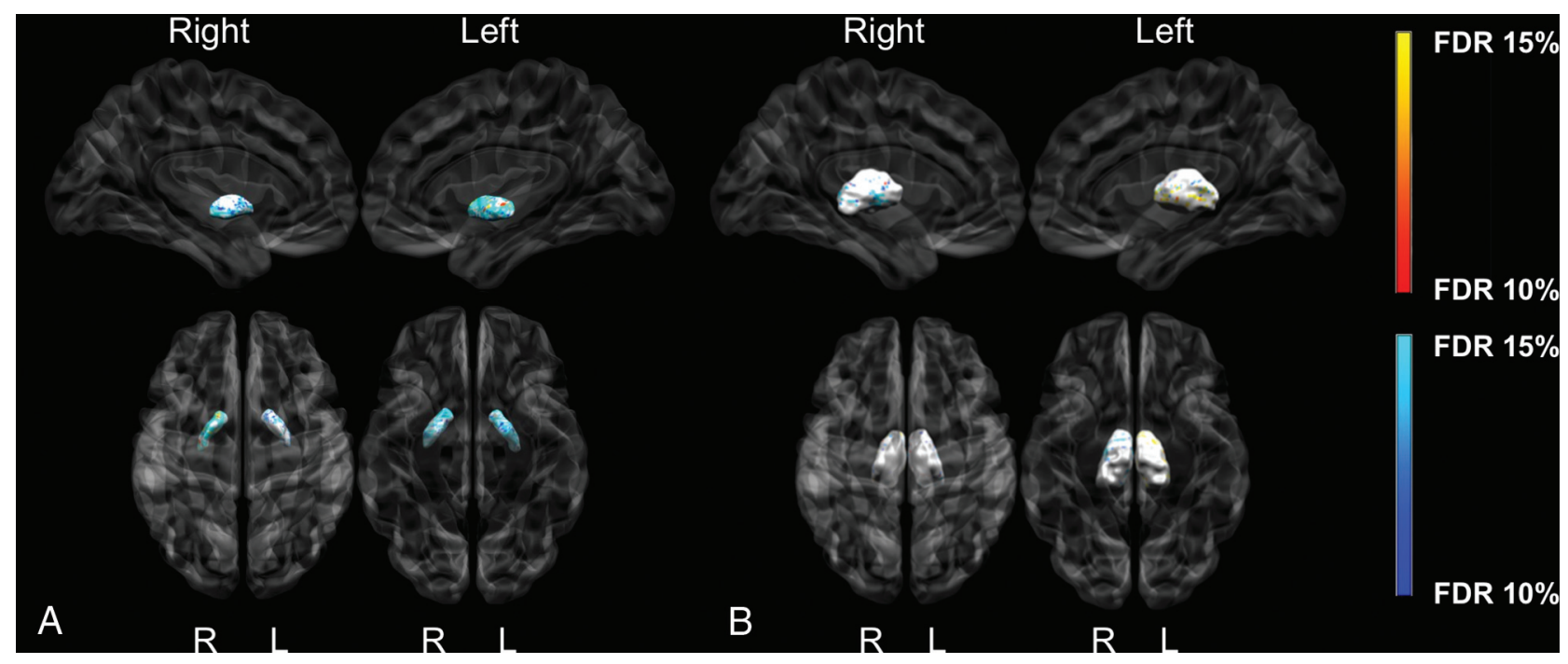

FIG 2. Group differences between CHD and control participants for the globus pallidus $(A)$ and thalamus surface area $(B)$. The darker blue colors indicate regions of significantly smaller surface area (more concave), and the darker orange indicates regions of significantly larger surface area (more convex) in CHD participants compared with controls $(q<0.15)$. Analyses were adjusted for total brain volume and socioeconomic status.

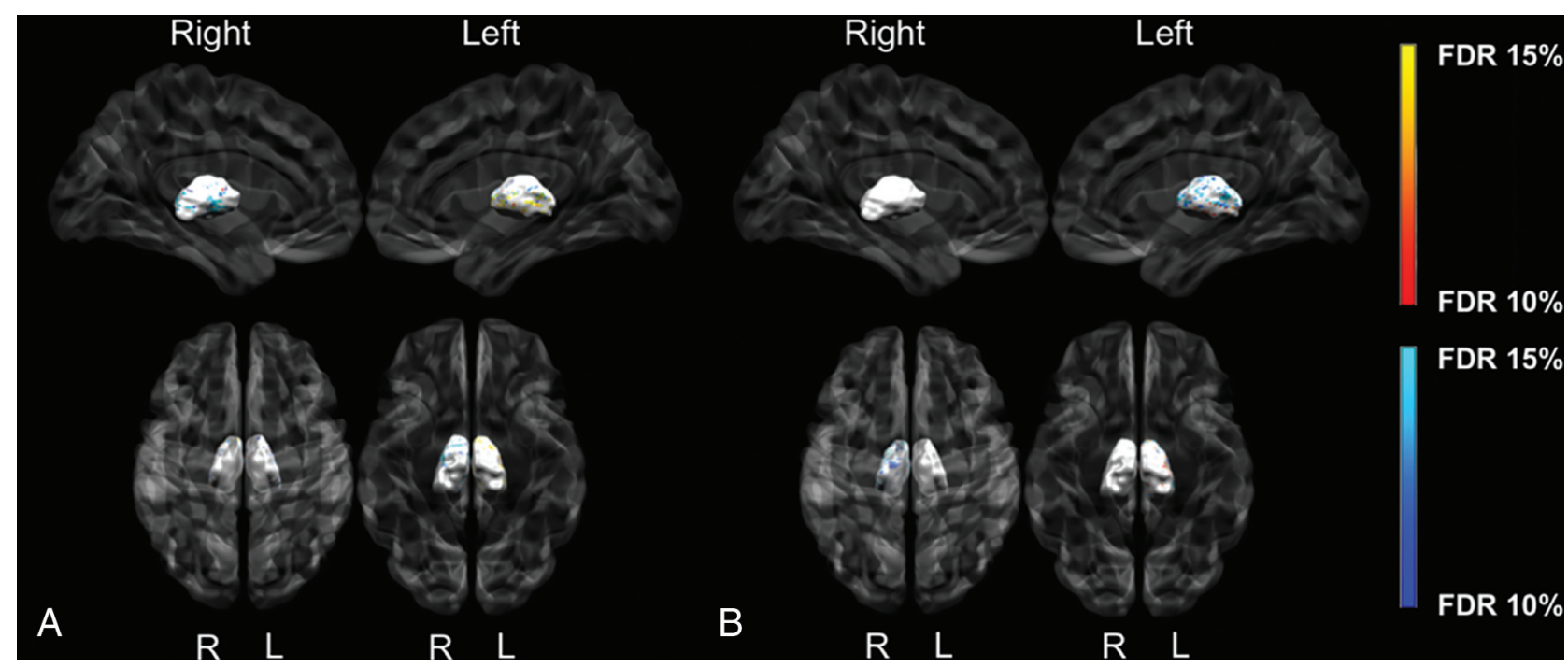

FIG 3. Group differences between CHD and control participants for globus pallidus $(A)$ and thalamus displacement (B). The darker blue colors indicate regions of significant inward displacement in CHD participants compared with controls. The darker orange colors indicate regions of significant outward displacement in CHD participants compared with controls $(q<0.15)$. Analyses were adjusted for total brain volume and socioeconomic status.

and the caudate, but differences remained nonsignificant $(q=$ 0.666-0.802). Volumes are reported in On-line Table 2. There were no significant differences between subcortical volumes of participants with or without brain abnormalities.

\section{DISCUSSION}

In the present study, we used quantitative MR imaging to comprehensively characterize the subcortical structures of youth with CHD. We found morphometric differences in the globus pallidus and thalamus, but not in the striatum of youth with CHD when compared to healthy peers. However, compared with controls, youth with CHD had only a smaller right globus pallidus, with no other volumetric differences. The lack of difference in volumes in our sample is consistent with previous studies that also did not find a significant difference in the overall basal ganglia volume of adolescents with CHD compared with controls after adjusting for total brain volume. ${ }^{9,10}$ Only Watson et al, ${ }^{9}$ in 2016, reported significantly smaller volumes in the bilateral caudate, which we did not observe in our volumetric analyses of the whole striatum or its subdivisions. This discrepancy in findings across studies may reside, in part, in the difference in the anatomic delineation of the subcortical structures used by each processing pipeline. Our results also support shape analyses being more sensitive to 
smaller regional differences because morphometry provides localized measures of surface area and displacement. ${ }^{7}$

Our findings are in line with the only previous study to apply voxel-based morphometric analyses in adolescents with CHD. Singh et $\mathrm{al}^{11}$ in 2018 , reported a widespread decreased proportion of gray matter tissue in many brain regions, including the thalamus and the subdivisions of the striatum. Indeed, we found both increased and decreased surface area across the lateral surfaces of the thalami bilaterally and inward and outward displacement on the surface of the left thalamus in the CHD group compared with controls. Similar widespread morphometric differences of the thalamus have been observed in individuals with mild cognitive impairment whose condition ultimately evolved to a diagnosis of Alzheimer disease. ${ }^{29}$ These findings are relevant because recent studies have reported a higher risk for cognitive decline in adults with CHD; however, this phenomenon is currently understudied. ${ }^{30}$

We detected a smaller surface area and inward displacement on the surface of the globus pallidus, which was concentrated anteriorly mainly on the lateral surfaces in the CHD group when compared to controls. Last, we found no differences in striatal morphometry between youth with $\mathrm{CHD}$ and healthy peers. Preliminary studies have found volumetric and morphometric differences of the striatum and globus pallidus in the context of addiction studies. ${ }^{31-33}$ However, we did not find any existing studies in relevant clinical populations with which we could compare our findings. Our findings highlight the need to conduct further structure-function studies to better understand the role of both the globus pallidus and the striatum in higher-order cognitive processes.

The development of subcortical structures has been shown to be highly heterogeneous; while regions of the basal ganglia are contracting with age, others are expanding. ${ }^{22}$ It is now well-established that brain development is often altered in fetuses and infants with CHD. ${ }^{34,35}$ The shape alterations of the subcortical structures observed in the current study could suggest a particular role for altered development of the subcortical gray matter. However, this hypothesis will need to be validated by a longitudinal study capturing early life development of the deep gray matter up until adulthood.

There are a few limitations worth considering in this study. First, we cannot generalize the findings of our study to a specific type of CHD because our sample is composed of a mixed cohort of CHD diagnoses. However, our inclusion of multiple types of $\mathrm{CHD}$ is a strength because we provide a range of CHD diagnoses that reflect the diversity found in the clinics. The higher socioeconomic status in our control group is likely caused by the recruitment of participants from local universities. We therefore included socioeconomic status as a covariate in all our analyses to control for it.

\section{CONCLUSIONS}

This study provides the first comprehensive evaluation of the subcortical structures of youth with CHD that includes morphometric and volumetric characterization of the globus pallidus, thalamus, and striatum using a hypothesis-driven approach. We report morphometric alterations in youth with $\mathrm{CHD}$ in the absence of volumetric reduction, which suggests that volume alone is not sufficient to detect and explain subtle structural differences in this clinical population. Morphometry is a valuable neuroradiologic tool in detecting subtle brain differences in the search for underlying mechanisms of brain functions. Nevertheless, studies with longitudinal follow-up are needed to better understand how these neuroanatomic differences may relate to functional outcomes in survivors of CHD.

\section{ACKNOWLEDGMENTS}

We thank the youth and their families for their participation in this study and the clinicians, technologists, and research assistants for their involvement in this study.

Disclosures: Christine Saint-Martin-UNRELATED: Board Membership: Societe de Radiologie du Quebec, Comments: member of the scientific committee. Guillaume Gilbert-UNRELATED: Employment: Philips Healthcare Canada, Comments: I work as a clinical scientist in MR imaging for Philips Healthcare Canada. Mallar Chakravarty-UNRELATED: Employment: McGill University.

\section{REFERENCES}

1. Marino BS, Lipkin PH, Newburger JW, et al; American Heart Association Congenital Heart Defects Committee, Council on Cardiovascular Disease in the Young, Council on Cardiovascular Nursing, and Stroke Council. Neurodevelopmental outcomes in children with congenital heart disease: evaluation and management: a scientific statement from the American Heart Association. Circulation 2012;126:1143-72 CrossRef Medline

2. Easson K, Dahan-Oliel N, Rohlicek C, et al. A comparison of developmental outcomes of adolescent neonatal intensive care unit survivors born with a congenital heart defect or born preterm. $J$ Pediatr 2019;207: 34-41.e2 CrossRef Medline

3. Latal B. Neurodevelopmental outcomes of the child with congenital heart disease. Clin Perinatol 2016;43:173-85 CrossRef Medline

4. Keir M, Ebert P, Kovacs AH, et al. Neurocognition in adult congenital heart disease: how to monitor and prevent progressive decline. Can J Cardiol 2019;35:1675-85 CrossRef Medline

5. Bolduc ME, Lambert H, Ganeshamoorthy S, et al. Structural brain abnormalities in adolescents and young adults with congenital heart defect: a systematic review. Dev Med Child Neurol 2018;60: 1209-24 CrossRef Medline

6. Voineskos AN, Winterburn JL, Felsky D, et al. Hippocampal (subfield) volume and shape in relation to cognitive performance across the adult lifespan. Hum Brain Mapp 2015;36:3020-37 CrossRef Medline

7. Chakravarty MM, Rapoport JL, Giedd JN, et al. Striatal shape abnormalities as novel neurodevelopmental endophenotypes in schizophrenia: a longitudinal study. Hum Brain Mapp 2015;36:1458-69 CrossRef Medline

8. Schuetze M, Park MT, Cho IY, et al. Morphological alterations in the thalamus, striatum, and pallidum in autism spectrum disorder. Neuropsychopharmacology 2016;41:2627-37 CrossRef Medline

9. Watson CG, Asaro LA, Wypij D, et al. Altered gray matter in adolescents with d-transposition of the great arteries. J Pediatr 2016; 169: 36-43 e1 CrossRef Medline

10. von Rhein M, Buchmann A, Hagmann C, et al. Brain volumes predict neurodevelopment in adolescents after surgery for congenital heart disease. Brain 2014;137:268-76 CrossRef Medline

11. Singh S, Kumar R, Roy B, et al. Regional brain gray matter changes in adolescents with single ventricle heart disease. Neurosci Lett 2018;665:156-62 CrossRef Medline

12. Fontes K, Rohlicek CV, Saint-Martin C, et al. Hippocampal alterations and functional correlates in adolescents and young adults 
with congenital heart disease. Hum Brain Mapp 2019;40:3548-60 CrossRef Medline

13. Delgado MR, Li J, Schiller D, et al. The role of the striatum in aversive learning and aversive prediction errors. Philos Trans $R$ Soc Lond, B, Biol Sci 2008;363:3787-800 CrossRef Medline

14. Gambaryan LS, Sarkisyan ZS. Role of the globus pallidus in the mechanisms of memory. Neurosci Behav Physiol Neuroscience Physiol 1983;13:470-75 CrossRef Medline

15. van Groen T, Kadish I, Wyss JM. The role of the laterodorsal nucleus of the thalamus in spatial learning and memory in the rat. Behav Brain Res 2002;136:329-37 CrossRef Medline

16. Hollingshead AB. Four Factor Index of Social Status [1975]. https:// ubir.buffalo.edu/xmlui/handle/10477/1879. Accessed September 15, 2019

17. Bedford SA, Park MT, Devenyi GA, et al; MRC AIMS Consortium. Large-scale analyses of the relationship between sex, age and intelligence quotient heterogeneity and cortical morphometry in autism spectrum disorder. Mol Psychiatry 2020;25:614-28 CrossRef Medline

18. Sadedin SP, Pope B, Oshlack A. Bpipe: a tool for running and managing bioinformatics pipelines. Bioinformatics 2012;28:1525-26 CrossRef Medline

19. Tustison NJ, Avants BB, Cook PA, et al. N4ITK: improved N3 bias correction. IEEE Trans Med Imaging 2010;29:1310-20 CrossRef Medline

20. Eskildsen SF, Coupé P, Fonov V, et al; Alzheimer's Disease Neuroimaging Initiative. BEaST: brain extraction based on nonlocal segmentation technique. Neuroimage 2012;59:2362-73 CrossRef Medline

21. Chakravarty MM, Steadman P, van Eede MC, et al. Performing label-fusion-based segmentation using multiple automatically generated templates. Hum Brain Mapp 2013;34:2635-54 CrossRef Medline

22. Raznahan A, Shaw PW, Lerch JP, et al. Longitudinal four-dimensional mapping of subcortical anatomy in human development. Proc Natl Acad Sci USA 2014;111:1592-97 CrossRef Medline

23. Shaw P, De Rossi P, Watson B, et al. Mapping the development of the basal ganglia in children with attention-deficit/hyperactivity disorder. J Am Acad Child Adolesc Psychiatry 2014;53:780-89.e11 CrossRef Medline
24. Tullo S, Devenyi GA, Patel R, et al. Warping an atlas derived from serial histology to 5 high-resolution MRIs. Sci Data 2018;5:180107 CrossRef Medline

25. Makowski C, Béland S, Kostopoulos P, et al. Evaluating accuracy of striatal, pallidal, and thalamic segmentation methods: comparing automated approaches to manual delineation. Neuroimage 2018; 170: 182-98 CrossRef Medline

26. Chris L, Gruner D, Groer L, et al. SciNet: lessons learned from building a power-efficient top-20 system and data centre. Journal of Physics: Conference Series 2010;256:012026

27. Lerch J. Voxel-wise morphometry using RMINC. 2010. https://rdrr.io/ github/Mouse-Imaging-Centre/RMINC/f/inst/documentation/RMINCuserGuide.pdf. Accessed September 15, 2019

28. The R Core Team. A Language and Environment for Statistical Computing. 2013. https://cran.r-project.org/doc/manuals/fullrefman. pdf. Accessed September 15, 2019

29. Kalin AM, Park MT, Chakravarty MM, et al. Subcortical shape changes, hippocampal atrophy and cortical thinning in future Alzheimer's disease patients. Front Aging Neurosci 2017;9:38 CrossRef Medline

30. Keir M, Ebert $\mathrm{P}$, Kovacs AH, et al. Neurocognition in adult congenital heart disease: how to monitor and prevent progressive decline. Can J Cardiol 2019;35:1675-85 CrossRef Medline

31. Garza-Villarreal EA, Chakravarty MM, Hansen B, et al. The effect of crack cocaine addiction and age on the microstructure and morphology of the human striatum and thalamus using shape analysis and fast diffusion kurtosis imaging. Transl Psychiatry 2017;7:e1122 CrossRef Medline

32. Janes AC, Park MT, Farmer S, et al. Striatal morphology is associated with tobacco cigarette craving. Neuropsychopharmacology 2015;40:406-11 CrossRef Medline

33. Smith MJ, Cobia DJ, Wang L, et al. Cannabis-related working memory deficits and associated subcortical morphological differences in healthy individuals and schizophrenia subjects. Schizophr Bull 2014;40:287-99 CrossRef Medline

34. Miller SP, McQuillen PS. Neurology of congenital heart disease: insight from brain imaging. Arch Dis Child Fetal Neonatal Ed 2007;92:F435-37 CrossRef Medline

35. Brossard-Racine M, du Plessis AJ, Vezina G, et al. Prevalence and spectrum of in utero structural brain abnormalities in fetuses with complex congenital heart disease. AJNR Am J Neuroradiol 2014; 35:1593-99 CrossRef Medline 